\title{
Sirtuin-4 (SIRT4), a therapeutic target with oncogenic and tumor-suppressive activity in cancer
}

This article was published in the following Dove Press journal:

OncoTargets and Therapy

\section{Guoyu Huang \\ Guanbao Zhu}

Department of Gastrointestinal Surgery, The First Affiliated Hospital, Wenzhou Medical University,

Wenzhou, People's Republic of China

\begin{abstract}
Several members of the sirtuin (SIRT) family, a highly conserved family of $\mathrm{NAD}^{+}$-dependent enzymes, have been shown to play a critical role in both promoting and/or suppressing tumorigenesis. In this study, recent progress in the field concerning SIRT4 and cancer was reviewed, and the relationship between SIRT4 and tumors was investigated. Subsequently, we evaluated the role of SIRT4 with oncogenic or tumor-suppressive activity in cancer, which may provide insight in identifying the underlying mechanism of action of SIRT4 in cancer. Finally, we explored the potential of SIRT4 as a therapeutic target in cancer therapy.
\end{abstract}

Keywords: cancer, genetic instability, metabolism, sirtuin, SIRT4

\section{Introduction}

The sirtuin (SIRT) family is a homologous analogue of the yeast silent information regulator (Sir2), which codes for histone deacetylation in mammals. ${ }^{1,2}$ Mammalian SIRTs are considered class III histone deacetylases, which are different from class I and II histone deacetylases. ${ }^{3,4}$ Seven members of the SIRT family (SIRT1-7) share a conserved catalytic domain. ${ }^{5}$ In addition to having a similar structure, members of the SIRT family have different enzymatic activities. ${ }^{6}$ For example, SIRT1-3 and SIRT7 are primarily lysine deacetylases, ${ }^{7,8}$ SIRT4 serves as both an ADP-ribosyl-transferase and lysine deacylase, ${ }^{9,10}$ SIRT5 serves as a lysine demalonylase, ${ }^{11}$ desuccinylase, ${ }^{12}$ and deglutarylase, ${ }^{13}$ and SIRT6 is an ADP-ribose transferase and deacetylase. ${ }^{14,15}$ In addition, the location of these proteins within the cell is different. ${ }^{6}$ SIRT1, SIRT6, and SIRT7 are located within the nucleus, SIRT2 is located in the cytoplasm, and SIRT3, SIRT4, and SIRT5 are located in the mitochondria. These differences in location suggested that different family members may have different functions. ${ }^{16}$ SIRT family members play an important role in stress resistance, genome stability, energy metabolism, and aging. ${ }^{5}$ Moreover, previous studies have shown that almost all members of the SIRT family play a role in tumorigenesis. ${ }^{6,17-19}$

Recent studies have demonstrated that members of the SIRT family often play opposite roles in tumor formation. ${ }^{6}$ For example, they can both promote and inhibit tumor formation. ${ }^{16,20,21}$ In previous studies, it was proposed to divide genes into oncogenes or tumor suppressor genes; however, only a small fraction of genes has been classified into these categories. $M y c$, for example, is an oncogene, ${ }^{22}$ whereas the $R B$ gene is a tumor suppressor gene. ${ }^{23}$ Other genes, including those of SIRT family members, have less specific roles, and the tumor promoting, or inhibiting, features of these genes may be dependent on the stage of tumor development, the tissue or organ microenvironment, and experimental conditions. ${ }^{24}$ For example, SIRT1 is highly expressed in gastric, ${ }^{25}$ colon, ${ }^{26}$ prostate,${ }^{27}$ and skin cancers,${ }^{28}$ as well as in several other tumors. This indicated
Correspondence: Guanbao Zhu Department of Gastrointestinal Surgery, The First Affiliated Hospital, Wenzhou Medical University, Nanbaixiang, Ouhai, Wenzhou, Zhejiang 325003, People's Republic of China Tel +86 57755579442 Fax +86 57755579445 Email zhuguanbao1958@gmail.com 
that SIRT1 may play a key role in promoting tumor formation in these malignant conditions. However, another study has demonstrated that expression of SIRT1 in human breast cancer tissue was reduced. ${ }^{29}$ Moreover, SIRT1 expression in a murine $\mathrm{APC}^{\mathrm{min} /+}$ model inhibited the formation of intestinal tumors. ${ }^{30}$ These observations were similar to the findings demonstrating that SIRT2 was downregulated in breast, ${ }^{31}$ glioma, and skin cancers. ${ }^{32,33}$ However, SIRT2 expression is enhanced in acute myeloid leukemia and prostate cancer. ${ }^{34,35}$ Therefore, the findings and conclusions of one tumor type cannot be extrapolated to that of another.

SIRT4 is a relatively understudied member of the SIRT family. At present, an increasing number of studies are focused on the correlation between SIRT4 and tumors, and these novel investigations will be reviewed in the following sections.

\section{The primary function of SIRT4}

SIRT4 is located within mitochondria ${ }^{9}$ and is widely distributed in adult and fetal tissues, as well as in adult thymus and white blood cells. ${ }^{36}$ Thus far, its most important function was related to regulating metabolism. ${ }^{4}$ Recent findings suggested that SIRT4 is active in nutrient-replete conditions, and deacetylates and inhibits malonyl CoA decarboxylase (MCD). MCD is an enzyme that produces acetyl CoA from malonyl CoA, which provides the carbon skeleton for lipogenesis and inhibits fat oxidation. ${ }^{37}$ Mice lacking SIRT4 showed increased MCD activity and deregulated lipid metabolism, resulting in increased exercise tolerance and protection against diet-induced obesity. ${ }^{37}$ This suggested that SIRT4 is an important regulator of lipid homeostasis. ${ }^{37}$ Moreover, in the liver, SIRT4 inhibition increased gene expression of mitochondrial and fatty acid metabolism-related enzymes, thereby suggesting that SIRT4 inhibition increased fat oxidative capacity. ${ }^{38}$ In addition, in pancreatic $\beta$-cells, SIRT4 repressed the activity of glutamate dehydrogenase (GDH) and subsequently downregulated insulin secretion in response to amino acids. ${ }^{9,39}$ Previous studies have shown that SIRT4 was essential when cells encounter toxic stress and nicotinamide phosphor-ribosyl-transferase, thereby helping maintain mitochondrial NAD levels. ${ }^{40}$ SIRT4 also inhibited the activity of pyruvate dehydrogenase, a key enzyme that affects the entrance of glucose into the tricarboxylic acid cycle. ${ }^{41}$ In a recent study, it was shown that SIRT4 is a protein deacetylase that removed 3 acyl moieties from lysine residues that were produced from leucine catabolism. Moreover, they showed that SIRT4-deficient mice had a dysregulated leucine metabolism that resulted in elevated insulin secretion. ${ }^{10}$
In addition to the regulatory role in metabolism, SIRT4 was found to have other activities. For example, SIRT4 regulated cell cycle progression and genomic fidelity in response to DNA damage. ${ }^{42}$ Camptothecin or ultraviolet (UV)-treated SIRT4 knockout mouse embryonic fibroblasts showed elevated cell death when compared to wild-type cells. ${ }^{42}$ In another study, it was demonstrated that SIRT4 reduced Drp1-driven mitochondrial fission. ${ }^{43}$ SIRT4 inhibited the phosphorylation of Drp1 and prevented Drp1 recruitment to the mitochondrial membrane by interacting with Fis- $1 .{ }^{43}$ Moreover, SIRT4 expression hampered activity of the MEK/ERK signaling pathway. ${ }^{43}$ Similar to other members of the SIRT family, SIRT4 may have complex biological functions that need to be further elucidated.

\section{The role of SIRT4 in tumors}

So far, most studies on SIRT4 in tumors have been performed on the role of SIRT4 as a tumor suppressor gene. In previous studies, it has been shown that overexpression of SIRT4 in vitro inhibited the proliferation of HeLa and $M y c$-driven human Burkitt lymphoma cells by inhibiting glutamine metabolism. ${ }^{42,44}$ Moreover, SIRT4 can inhibit the proliferation of RKO,${ }^{45}$ HT29, SW480, HCT116, ${ }^{46}$ DLD-1, H1299, A549, and DU145 cells. ${ }^{43,47}$ Overexpression of SIRT4 inhibited the migration and invasion of SW480, HCT116, H1299, and A549 cells. ${ }^{43,46}$ Knockdown of SIRT4 increased the migration ability of KYSE170, H1299, and A549 cells. ${ }^{43,48}$ In vivo studies in nude mice have shown that knockout of SIRT4 in mouse embryonic fibroblast cells forms larger tumors. ${ }^{42,45,47}$ Moreover, SIRT4 knockout mice spontaneously develop lung cancer, liver cancer, breast cancer, and lymphomas. ${ }^{42}$ In a $\mathrm{Tsc} 2^{-/-}$murine embryonic fibroblast xenograft model, overexpression of SIRT4 reduced cell proliferation and transformation and delayed tumor development. ${ }^{47}$ In addition, SIRT4 overexpression resulted in a significant decrease in RKO tumor size in nude mice. ${ }^{45}$ The Cancer Genome Atlas microarray database analysis indicated that at the mRNA level, SIRT4 mRNA levels in human breast, bladder, gastric, colon, thyroid, and ovarian cancers were decreased when compared with normal tissues. ${ }^{42,47}$ Moreover, RT-PCR analysis of mRNA extracted from human tissue demonstrated that SIRT4 mRNA levels in colon, ${ }^{45}$ breast, ${ }^{49}$ endometrial, ${ }^{50}$ and esophageal cancers were reduced. ${ }^{48}$ Decreased SIRT4 protein levels in gastric, colon, liver, lung, and esophageal cancer tissues were associated with worse pathological grading and other clinicopathological parameters. ${ }^{43,45,46,51,52}$ At the same time, reduced SIRT4 protein levels correlated with poor prognosis in colon, lung, and esophageal cancer. ${ }^{43,45,46,48}$ 
Table I Summary of the roles of SIRT4 in tumors

\begin{tabular}{|c|c|c|c|}
\hline Phenotype & Tumor types & Role of SIRT4 & References \\
\hline SIRT4 protein levels are increased & Breast and esophageal cancer & Cancer promoting & 54,55 \\
\hline SIRT4 protein levels are decreased & $\begin{array}{l}\text { Gastric, colorectal, liver, lung, endometrial, } \\
\text { and esophageal cancer }\end{array}$ & Cancer suppressing & $\begin{array}{l}42,43,45,46 \\
48,51,52,65\end{array}$ \\
\hline SIRT4 overexpression inhibits cell proliferation & $\begin{array}{l}\text { Myc-induced B cell lymphoma, colorectal } \\
\text { cancer, cervical lung, and esophageal cancer }\end{array}$ & Cancer suppressing & $42-46$ \\
\hline SIRT4 knockout mice spontaneously develop cancers & Lung, liver, breast cancer, and lymphoma & Cancer suppressing & 42 \\
\hline $\begin{array}{l}\text { SIRT4 overexpression increases the survival rate of } \\
\text { cancer cells in DNA-damaging conditions }\end{array}$ & HepG2 cells & Cancer promoting & 53 \\
\hline
\end{tabular}

Abbreviation: SIRT4, Sirtuin-4.

Previous studies have also shown that SIRT4 may not be just a tumor suppressor gene. ${ }^{53-55}$ For example, Shi et al ${ }^{55}$ evaluated 241 cases of paired breast cancer and noncancerous tissues by immunohistochemical staining and found that $48(19.92 \%)$ tumor tissue samples were SIRT4 positive, whereas only $29(12.03 \%)$ adjacent nontumor tissue samples were SIRT4 positive. ${ }^{55}$ In addition, studies were performed using tissue microarray staining, which included 93 cases of esophageal carcinoma and adjacent nontumor tissues of the esophagus, and showed that the SIRT4 protein level in esophageal cancer tissue was higher compared to that in adjacent nontumor esophageal tissue. The mean survival time of esophageal cancer patients who demonstrated higher levels of SIRT4 protein was lower compared to that of patients who demonstrated lower level of SIRT4 (26.2 vs 32.1 months). Although trending, the data were not significantly different. ${ }^{54}$ At the cellular level, SIRT4 expression was increased under conditions of DNA damage, including camptothecin, UV, and oncogene-induced transformation. Moreover, SIRT4 overexpression increased the survival rate of HepG2 cells in DNA-damaging conditions, reduced mortality, and SIRT4 loss sensitized cells after drug treatment. ${ }^{53}$ Taken together, these studies demonstrated that SIRT4 is upregulated in several tumors, can regulate oncogenic stress, and prepare tumor cells to gain selective advantages. Therefore, in several tumors and cases, SIRT4 may play an oncogenic role, which merits further studies for confirmation (Table 1).

\section{Two possible mechanisms for the role of SIRT4 in tumors Inhibiting metabolism}

Both tumorigenic and normal cells have distinct metabolic patterns, and an altered energy metabolism is a key characteristic of tumors. ${ }^{24}$ Tumor cells often appear to enhance both the glucose and glutamine metabolism to provide the energy that is required for tumor growth. ${ }^{56,57}$ Due to the higher degree of malignancy, the proliferation rate may be faster; therefore, the corresponding energy demand may be higher. Previous studies on SIRT4 indicated that SIRT4 inhibited tumor metabolism, particularly the glutamine metabolism, ${ }^{41,42,47}$ suggesting that SIRT4 may function as a tumor suppressor. SIRT4 is also thought to be a keeper of cellular energy metabolism. ${ }^{41}$ SIRT4 can be inhibited by mTOR and CtBP, and subsequently, through the inhibition of GDH, inhibit the glutamine reflux. A lower level of SIRT4 will increase the cellular glutamine reflux and promote tumor formation. ${ }^{42,47,58}$ Overexpression of SIRT4 can inhibit glutamine consumption and ammonia production in $M y c$-induced B cell lymphoma. In addition, Ramos and Raji cells inhibit the GDH activity in colon and esophageal cancer cells in vitro. ${ }^{44,46,48}$

An enhanced glucose metabolism is a feature of tumors, ${ }^{24}$ and Jeong et $\mathrm{a}^{142}$ demonstrated that SIRT4 inhibited tumor growth by inhibiting the mitochondrial glutamine metabolism. ${ }^{47}$ However, an effect of SIRT4 on the glucose metabolism was not observed. Recent study has shown that SIRT4 had inhibitory effects on pyruvate dehydrogenase. ${ }^{41}$ Because pyruvate dehydrogenase is a key enzyme that affects the entrance of glucose into the tricarboxylic acid cycle, it was suggested that SIRT4 may play a role in the glucose metabolism. In our previous study, we demonstrated that SIRT4 increased mortality in colorectal cancer cells that were grown in glucose-deprived culture media and that addition of glutamine to a downstream metabolite, dimethyl $\alpha$-ketoglutarate, could counteract this effect. ${ }^{45} \mathrm{We}$ also found that overexpression of SIRT4 reduced the survival rate of colorectal cancer cells in glutaminedeprived media. ${ }^{45}$ These findings were in line with the notion that SIRT4 inhibited the glutamine metabolism and indicated that SIRT4 inhibited the glucose metabolism in colorectal cancer cells. ${ }^{45}$ So far, there was no consistent conclusion about whether SIRT4 affected the glucose metabolism or not.

\section{Regulation of genome stability}

DNA damage causes cell cycle arrest, which is one way for cells to maintain genome stability. ${ }^{59}$ Glutamine is a key 
substance of cells that transit from the G1 phase into the $\mathrm{S}$ phase. ${ }^{60}$ In a previous study, it was shown that SIRT4 expression increased in DNA-damaging conditions, leading to cell cycle arrest by inhibiting the glutamine reflux, thereby reducing the accumulation of damage to DNA, and promoting self-protection. ${ }^{42}$ Therefore, decreased SIRT4 expression in nontumor cells resulted in accumulation of cell mutations while the DNA is damaged, which contributed to tumor formation. ${ }^{42}$ In this setting, SIRT4 in nontumor cells may have a tumor suppressor role in DNA-damaging conditions. This mechanism may also be established in tumor cells. In 1 study, it was found that SIRT4 was increased in HepG 2 cells after treatment with cisplatin, radiation, and UV irradiation. ${ }^{53}$ In addition, overexpression of SIRT4 reduced the death rate of HepG2 cells in DNA-damaging conditions, thereby increasing the cellular clone formation rate after radiation treatment. ${ }^{53}$ Therefore, in regulating genome stability, SIRT4 plays a dual role in inhibiting tumor formation and protection.

In summary, in nontumor cells, SIRT4 protected against the accumulation of DNA damage, reduced cell death in DNA-damaging conditions, and acted as a tumor suppressor. However, in tumor cells, SIRT4 played a dual role. First, in normal conditions, SIRT4 inhibited the glutamine metabolism to reduce tumor metabolism, which inhibited tumor proliferation. In extreme DNA-damaging conditions, such as in the presence of chemotherapeutic drugs, SIRT4 protected tumor cells, thereby acting as an oncogene. Simultaneously, SIRT4 reduced tumor cell evolution, delayed tumor cell mutation accumulation, and acted as a tumor suppressor. For tumor cells, mutation accumulation is more important than temporary existence, and therefore, SIRT4 may generally act as a tumor suppressor.

\section{Can SIRT4 be a therapeutic target for cancer? \\ Synergistic glucose metabolism inhibitor}

Therapeutics that can block tumor cells in their metabolic pathway are currently under development as novel tumor treatment strategies. ${ }^{61,62}$ For example, targeting the glucose metabolism pathway using glucose inhibitors has been proposed in tumor therapy. ${ }^{61-63}$ However, by activating additional metabolic pathways, including the glutamine metabolism, tumor cells can survive with a reduced glucose metabolism. Thus, the mitochondrial glutamine metabolism can compensate for the lack of glucose and recharge the mitochondrial tricarboxylic acid cycle. ${ }^{31,34}$ Therefore, blocking glutamine metabolism, and simultaneously blocking glucose and glutamine metabolism, has great potential in cancer therapy. ${ }^{64}$ In our previous study, we demonstrated that colorectal cancer cells maintained growth in a glucose-deprived environment, and SIRT4 overexpression increased death resulting from glucose deprivation in colorectal cancer. ${ }^{45}$ Moreover, synergistic treatment by SIRT4 overexpression and glucose inhibitors significantly increases colorectal cancer cell death. ${ }^{45}$ Overexpression of SIRT4 increased the sensitivity of Burkitt lymphoma cells to glucose deprivation and increased cell death that was associated with glucose metabolism inhibitor treatment. ${ }^{44}$ Thus, these findings indicated the therapeutic potential of the SIRT4 targeting metabolism, particularly its potential for the treatment of tumors in combination with a glucose metabolism inhibitor.

\section{Synergistic chemotherapy drugs}

Previous study has shown that SIRT4 delayed the cell cycle in DNA-damaging conditions. ${ }^{42}$ Chemotherapy drugs can damage DNA; however, when used in the clinic, several have reported that SIRT4 increased the sensitivity of tumor cells to chemotherapeutic drugs. In our previous study, we showed that 5-FU increased the sensitivity of colorectal cancer cells to chemotherapy drugs by delaying the cell cycle. ${ }^{45}$ In another study, it was reported that overexpression of SIRT4 reduced the mortality rate of cisplatin and increased the rate of clone formation. ${ }^{53}$ To some extent, these 2 studies were not contradictory because SIRT4 reduced the viability of tumor cells in the presence of chemotherapy drugs by delaying the cell cycle. Moreover, it may help tumor cells prevent the DNA damage caused by chemotherapeutics, allowing them to escape death, leading to a higher survival rate. As discussed above, chemotherapy-induced DNA damage can both kill tumor cells and help them survive, such that they acquire more mutations and become more aggressive. Therefore, the use of SIRT4 in antitumor therapy merits further investigation, especially to confirm that it inhibits proliferation activity while increasing the survival rate.

\section{Conclusion}

SIRT4 may act as an oncogene and tumor suppressor gene, which may be dependent on the affected tissue types and specific tumor environment. SIRT4 showed enhanced potential as an antitumor therapeutic when used in synergy with glucose metabolism inhibitors. However, the use of SIRT4 in combination with cytotoxic chemotherapy drugs requires further investigation. Additionally, particular in vivo studies are warranted. In addition, it would be worth exploring the underlying mechanism of action and regulatory effect of SIRT4 on the glucose metabolism, the relationship between 
SIRT4 and both the epithelial to mesenchymal transition and autophagy, as well as the impact of SIRT3, which is also located in mitochondria.

\section{Acknowledgment}

This study was financially supported by the Project of Wenzhou Science and Technology Bureau (No Y20160404) and Zhejiang Natural Science Foundation (No LY18H160055).

\section{Disclosure}

The authors report no conflicts of interest in this work.

\section{References}

1. Imai S, Armstrong CM, Kaeberlein M, Guarente L. Transcriptional silencing and longevity protein Sir2 is an NAD-dependent histone deacetylase. Nature. 2000;403(6771):795-800.

2. Frye RA. Phylogenetic classification of prokaryotic and eukaryotic Sir2like proteins. Biochem Biophys Res Commun. 2000;273(2):793-798.

3. Saunders LR, Verdin E. Cell biology. Stress response and aging. Science. 2009;323(5917):1021-1022.

4. Zhu Y, Yan Y, Principe DR, Zou X, Vassilopoulos A, Gius D. SIRT3 and SIRT4 are mitochondrial tumor suppressor proteins that connect mitochondrial metabolism and carcinogenesis. Cancer Metab. 2014;2:15.

5. Finkel T, Deng CX, Mostoslavsky R. Recent progress in the biology and physiology of sirtuins. Nature. 2009;460(7255):587-591.

6. Roth M, Chen WY. Sorting out functions of sirtuins in cancer. Oncogene. 2014;33(13):1609-1620

7. Feldman JL, Baeza J, Denu JM. Activation of the protein deacetylase SIRT 6 by long-chain fatty acids and widespread deacylation by mammalian sirtuins. J Biol Chem. 2013;288(43):31350-31356.

8. Madsen AS, Andersen C, Daoud M, et al. Investigating the sensitivity of $\mathrm{NAD}^{+}$-dependent sirtuin deacylation activities to NADH. J Biol Chem. 2016;291(13):7128-7141.

9. Haigis MC, Mostoslavsky R, Haigis KM, et al. SIRT4 inhibits glutamate dehydrogenase and opposes the effects of calorie restriction in pancreatic beta cells. Cell. 2006;126(5):941-954.

10. Anderson KA, Huynh FK, Fisher-Wellman K, et al. SIRT4 is a lysine deacylase that controls leucine metabolism and insulin secretion. Cell Metab. 2017;25(4):838.e15-855.e15.

11. Peng $C, L u Z$, Xie Z, et al. The first identification of lysine malonylation substrates and its regulatory enzyme. Mol Cell Proteomics. 2011;10(12):M111.012658.

12. Du J, Zhou Y, Su X, et al. Sirt5 is a NAD-dependent protein lysine demalonylase and desuccinylase. Science. 2011;334(6057):806-809.

13. Tan M, Peng C, Anderson KA, et al. Lysine glutarylation is a protein posttranslational modification regulated by SIRT5. Cell Metab. 2014;19(4):605-617.

14. Michishita E, McCord RA, Berber E, et al. SIRT6 is a histone H3 lysine 9 deacetylase that modulates telomeric chromatin. Nature. 2008; 452(7186):492-496.

15. Mostoslavsky R, Chua KF, Lombard DB, et al. Genomic instability and aging-like phenotype in the absence of mammalian SIRT6. Cell. 2006;124(2):315-329.

16. Haigis MC, Sinclair DA. Mammalian sirtuins: biological insights and disease relevance. Annu Rev Pathol. 2010;5:253-295.

17. Martinez-Pastor B, Mostoslavsky R. Sirtuins, metabolism, and cancer. Front Pharmacol. 2012;3:22.

18. Carafa V, Rotili D, Forgione M, et al. Sirtuin functions and modulation: from chemistry to the clinic. Clin Epigenetics. 2016;8:61.

19. George J, Ahmad N. Mitochondrial sirtuins in cancer: emerging roles and therapeutic potential. Cancer Res. 2016;76(9):2500-2506.
20. Yuan H, Su L, Chen WY. The emerging and diverse roles of sirtuins in cancer: a clinical perspective. Onco Targets Ther. 2013;6:1399-1416.

21. Guarente L. The many faces of sirtuins: Sirtuins and the Warburg effect. Nat Med. 2014;20(1):24-25.

22. Hoffman B, Liebermann DA. The proto-oncogene c-myc and apoptosis. Oncogene. 1998;17(25):3351-3357.

23. Dick FA, Rubin SM. Molecular mechanisms underlying RB protein function. Nat Rev Mol Cell Biol. 2013;14(5):297-306.

24. Hanahan D, Weinberg RA. Hallmarks of cancer: the next generation. Cell. 2011;144(5):646-674.

25. Cha EJ, Noh SJ, Kwon KS, et al. Expression of DBC1 and SIRT1 is associated with poor prognosis of gastric carcinoma. Clin Cancer Res. 2009;15(13):4453-4459.

26. Stunkel W, Peh BK, Tan YC, et al. Function of the SIRT1 protein deacetylase in cancer. Biotechnol J. 2007;2(11):1360-1368.

27. Huffman DM, Grizzle WE, Bamman MM, et al. SIRT1 is significantly elevated in mouse and human prostate cancer. Cancer Res. 2007;67(14): $6612-6618$

28. Hida Y, Kubo Y, Murao K, Arase S. Strong expression of a longevityrelated protein, SIRT1, in Bowen's disease. Arch Dermatol Res. 2007; 299(2):103-106.

29. Wang RH, Sengupta K, Li C, et al. Impaired DNA damage response, genome instability, and tumorigenesis in SIRT1 mutant mice. Cancer Cell. 2008;14(4):312-323.

30. Firestein R, Blander G, Michan S, et al. The SIRT1 deacetylase suppresses intestinal tumorigenesis and colon cancer growth. PLoS One. 2008;3(4):e2020.

31. Kim HS, Vassilopoulos A, Wang RH, et al. SIRT2 maintains genome integrity and suppresses tumorigenesis through regulating APC/C activity. Cancer Cell. 2011;20(4):487-499.

32. Hiratsuka M, Inoue T, Toda T, et al. Proteomics-based identification of differentially expressed genes in human gliomas: down-regulation of SIRT2 gene. Biochem Biophys Res Commun. 2003;309(3):558-566.

33. Ming M, Qiang L, Zhao B, He YY. Mammalian SIRT2 inhibits keratin 19 expression and is a tumor suppressor in skin. Exp Dermatol. 2014; 23(3):207-209.

34. Dan L, Klimenkova O, Klimiankou M, et al. The role of sirtuin 2 activation by nicotinamide phosphoribosyltransferase in the aberrant proliferation and survival of myeloid leukemia cells. Haematologica. 2012;97(4):551-559.

35. Hou H, Chen W, Zhao L, et al. Cortactin is associated with tumour progression and poor prognosis in prostate cancer and SIRT2 other than HADC6 may work as facilitator in situ. J Clin Pathol. 2012;65(12):1088-1096.

36. Frye RA. Characterization of five human cDNAs with homology to the yeast SIR2 gene: Sir2-like proteins (sirtuins) metabolize NAD and may have protein ADP-ribosyltransferase activity. Biochem Biophys Res Commun. 1999;260(1):273-279.

37. Laurent G, German NJ, Saha AK, et al. SIRT4 coordinates the balance between lipid synthesis and catabolism by repressing malonyl CoA decarboxylase. Mol Cell. 2013;50(5):686-698.

38. Nasrin N, Wu X, Fortier E, et al. SIRT4 regulates fatty acid oxidation and mitochondrial gene expression in liver and muscle cells. $J$ Biol Chem. 2010;285(42):31995-32002.

39. Argmann C, Auwerx J. Insulin secretion: SIRT4 gets in on the act. Cell. 2006;126(5):837-839.

40. Yang H, Yang T, Baur JA, et al. Nutrient-sensitive mitochondrial NAD levels dictate cell survival. Cell. 2007;130(6):1095-1107.

41. Mathias RA, Greco TM, Oberstein A, et al. Sirtuin 4 is a lipoamidase regulating pyruvate dehydrogenase complex activity. Cell. 2014; 159(7):1615-1625.

42. Jeong SM, Xiao C, Finley LW, et al. SIRT4 has tumor-suppressive activity and regulates the cellular metabolic response to DNA damage by inhibiting mitochondrial glutamine metabolism. Cancer Cell. 2013; 23(4):450-463.

43. Fu L, Dong Q, He J, et al. SIRT4 inhibits malignancy progression of NSCLCs, through mitochondrial dynamics mediated by the ERK-Drp1 pathway. Oncogene. 2017;36(19):2724-2736. 
44. Jeong SM, Lee A, Lee J, Haigis MC. SIRT4 suppresses tumor formation in genetic models of Myc-induced B cell lymphoma. J Biol Chem. 2013;289(7):4135-4144.

45. Huang G, Cheng J, Yu F, et al. Clinical and therapeutic significance of sirtuin-4 expression in colorectal cancer. Oncol Rep. 2016;35(5): 2801-2810.

46. Miyo M, Yamamoto H, Konno M, et al. Tumour-suppressive function of SIRT4 in human colorectal cancer. Br J Cancer. 2015;113(3):492-499.

47. Csibi A, Fendt SM, Li C, et al. The mTORC1 pathway stimulates glutamine metabolism and cell proliferation by repressing SIRT4. Cell. 2013;153(4):840-854.

48. Nakahara Y, Yamasaki M, Sawada G, et al. Downregulation of SIRT4 expression is associated with poor prognosis in esophageal squamous cell carcinoma. Oncology. 2016;90(6):347-355.

49. Igci M, Kalender ME, Borazan E, et al. High-throughput screening of Sirtuin family of genes in breast cancer. Gene. 2016;586(1):123-128.

50. Bartosch C, Monteiro-Reis S, Almeida-Rios D, et al. Assessing sirtuin expression in endometrial carcinoma and non-neoplastic endometrium. Oncotarget. 2016;7(2):1144-1154.

51. Huang G, Lai X, Chen Z, et al. Sirtuin-4 (SIRT4) is downregulated in hepatocellular carcinoma and associated with clinical stage. Int J Clin Exp Pathol. 2016;9(6):6511-6517.

52. Huang G, Cui F, Yu F, et al. Sirtuin-4 (SIRT4) is downregulated and associated with some clinicopathological features in gastric adenocarcinoma. Biomed Pharmacother. 2015;72:135-139.

53. Jeong SM, Hwang S, Seong RH. SIRT4 regulates cancer cell survival and growth after stress. Biochem Biophys Res Commun. 2016;470(2): 251-256.

54. Lai X, Yu Z, Chen X, Huang G. SIRT4 is upregulated in Chinese patients with esophageal cancer. Int J Clin Exp Pathol. 2016;9(10): 10543-10549.
55. Shi Q, Liu T, Zhang X, et al. Decreased sirtuin 4 expression is associated with poor prognosis in patients with invasive breast cancer. Oncol Lett. 2016;12(4):2606-2612.

56. Daye D, Wellen KE. Metabolic reprogramming in cancer: unraveling the role of glutamine in tumorigenesis. Semin Cell Dev Biol. 2012; 23(4):362-369.

57. Tennant DA, Duran RV, Gottlieb E. Targeting metabolic transformation for cancer therapy. Nat Rev Cancer. 2010;10(4):267-277.

58. Wang L, Zhou H, Wang Y, Cui G, Di LJ. CtBP maintains cancer cell growth and metabolic homeostasis via regulating SIRT4. Cell Death Dis. 2015;6:e1620.

59. Kültz D. Molecular and evolutionary basis of the cellular stress response. Annu Rev Physiol. 2005;67:225-257.

60. Colombo SL, Palacios-Callender M, Frakich N, et al. Molecular basis for the differential use of glucose and glutamine in cell proliferation as revealed by synchronized HeLa cells. Proc Natl Acad Sci US A. 2011; 108(52):21069-21074.

61. Zhao Y, Butler EB, Tan M. Targeting cellular metabolism to improve cancer therapeutics. Cell Death Dis. 2013;4:e532.

62. Galluzzi L, Kepp O, Vander Heiden MG, Kroemer G. Metabolic targets for cancer therapy. Nat Rev Drug Discov. 2013;12(11):829-846.

63. Ahmad IM, Abdalla MY, Aykin-Burns N, et al. 2-Deoxyglucose combined with wild-type p53 overexpression enhances cytotoxicity in human prostate cancer cells via oxidative stress. Free Radic Biol Med. 2008;44(5):826-834.

64. Le A, Lane AN, Hamaker M, et al. Glucose-independent glutamine metabolism via TCA cycling for proliferation and survival in B cells. Cell Metab. 2012;15(1):110-121.

65. Chen X, Lai X, Wu C, et al. Decreased SIRT4 protein levels in endometrioid adenocarcinoma tissues are associated with advanced AJCC stage. Cancer Biomark. 2017;19(4):419-424.
OncoTargets and Therapy

\section{Publish your work in this journal}

OncoTargets and Therapy is an international, peer-reviewed, open access journal focusing on the pathological basis of all cancers, potential targets for therapy and treatment protocols employed to improve the management of cancer patients. The journal also focuses on the impact of management programs and new therapeutic agents and protocols on

\section{Dovepress}

patient perspectives such as quality of life, adherence and satisfaction The manuscript management system is completely online and includes a very quick and fair peer-review system, which is all easy to use. Visit http://www.dovepress.com/testimonials.php to read real quotes from published authors. 